\title{
A 4.5 MGy TID-Tolerant CMOS Bandgap Reference Circuit Using a Dynamic Base Leakage Compensation Technique
}

\author{
Ying Cao, Student Member, IEEE, Wouter De Cock, Michiel Steyaert, Fellow, IEEE, and
} Paul Leroux, Senior Member, IEEE

\begin{abstract}
The total-ionizing-dose (TID) radiation tolerance of bandgap references in deep-submicron CMOS technology is generally limited by the radiation introduced leakage current in diodes. An analysis of this phenomenon is given in this paper, and a dynamic base leakage compensation (DBLC) technique is proposed to improve the radiation hardness of a bandgap reference built in a standard $0.13 \mu \mathrm{m}$ CMOS technology. A temperature coefficient of $15 \mathrm{ppm} /{ }^{\circ} \mathrm{C}$ from $-40^{\circ} \mathrm{C}$ to $125^{\circ} \mathrm{C}$ is measured before irradiation. The voltage variation from $0^{\circ} \mathrm{C}$ to $100^{\circ} \mathrm{C}$ is only $\pm 1 \mathrm{mV}$ for an output voltage of $600 \mathrm{mV}$. Gamma irradiation assessment proves that the bandgap reference is tolerant to a total ionizing dose of at least 4.5 MGy. The output reference voltage exhibits a variation of less than $3 \%$ during the entire experiment, when the chip is irradiated by gamma ray at a dose rate of $27 \mathrm{kGy} / \mathrm{h}$.
\end{abstract}

Index Terms-Bandgap, dynamic base leakage compensation, low-voltage, radiation-hardened, total ionizing dose.

\section{INTRODUCTION}

$\mathbf{R}$ EFERENCE voltage generators are critical building blocks in many analog/mixed-signal systems such as $\mathrm{A} / \mathrm{D}, \mathrm{D} / \mathrm{A}$ converters and voltage regulators. The generators are required to be stabilized over process, supply voltage, and temperature (PVT) variations. For applications in high-energy physics, space and nuclear reactors, the reference voltage also needs to be stable under high ionizing radiation dose. The CMOS bandgap reference technique [1] is one of the most popular solutions which has been successfully implemented to achieve the requirements. However, such a bandgap reference can only provide a fixed voltage of $1.25 \mathrm{~V}$, which limits the low supply voltage (e.g., sub-1-V) operation coming along with the CMOS technology downscaling.

Manuscript received September 23, 2012; revised December 07, 2012; accepted December 07, 2012. Date of publication February 01, 2013; date of current version August 14, 2013. This work was carried out at the ESAT-MICAS laboratory, KU Leuven, and supported by SCK-CEN, the Belgian Nuclear Research Centre.

Y. Cao is with the ESAT-MICAS division, KU Leuven, B-3001 Heverlee, Belgium and also with SCK-CEN, the Belgian Nuclear Research Centre, B-2400 Mol, Belgium (e-mail: ying.cao@esat.kuleuven.be).

W. De Cock is with SCK-CEN, the Belgian Nuclear Research Centre, B-2400 Mol, Belgium (e-mail: wdcock@sckcen.be).

M. Steyaert is with the ESAT-MICAS division, KU Leuven, B-3001 Heverlee, Belgium (e-mail: mihciel.steyaert@esat.kuleuven.be).

P. Leroux is with the ICT-RELIC division, Katholieke Hogeschool Kempen, B-2440 Geel, Belgium and also with ESAT-MICAS division, KU Leuven, B-3001 Heverlee, Belgium (e-mail: paul.leroux@khk.be).

Color versions of one or more of the figures in this paper are available online at http://ieeexplore.ieee.org.

Digital Object Identifier 10.1109/TNS.2012.2233755

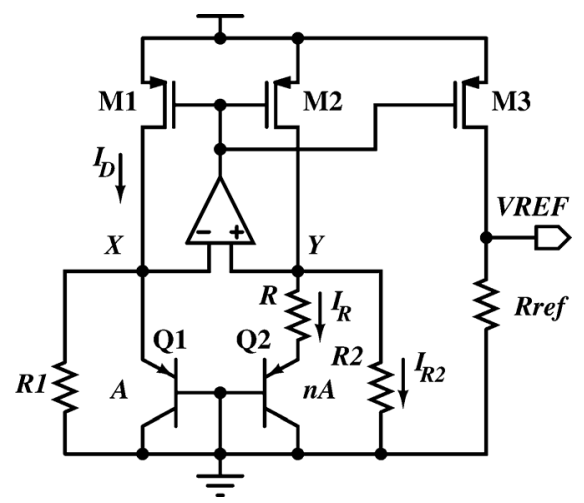

Fig. 1. A current-mode CMOS bandgap reference with sub-1-V operation.

As a mainstream integrated circuit fabricating process, commercial CMOS technology has been successfully implemented under ionizing radiation up to $1 \mathrm{MGy}$, by laying out the NMOS transistors in enclosed geometry [2]. Recent research also shows a trend in advanced CMOS technologies toward increased total dose hardness, due to downscaling of the CMOS gate oxide thickness [3]. This makes modern deep-submicron CMOS technology more suitable for radiation tolerant design. However, it has been found that conventional bandgap references in submicron CMOS technology are rather vulnerable to total-ionizingdose (TID) effect [4], due to radiation damage in the diodes.

In this work, a radiation-hardened bandgap reference with sub-1-V operation is designed in standard $0.13 \mu \mathrm{m}$ CMOS technology. An analysis on the TID effects in CMOS bandgap references is given. In order to achieve MGy-level ionizing radiation tolerance, a dynamic base leakage compensation (DBLC) technique is proposed to eliminate the radiation introduced leakage current in the bandgap core. The remainder of the paper is organized as follows. An analysis on the TID effects in conventional low-voltage CMOS bandgap references is given in Section II. Section III explains the proposed DBLC technique in detail. Pre-rad measurement and radiation assessment results are discussed in Section IV. In Section V, a conclusion is drawn.

\section{TOTAL IONIZING Dose EFFects IN CMOS BANDGAP REFERENCES}

\section{A. CMOS Bandgap Reference With Sub-1-V Operation}

A CMOS bandgap reference circuit with sub-1-V operation can be built in a current-mode structure as shown in Fig. 1 [5]. A temperature independent current is first generated by 


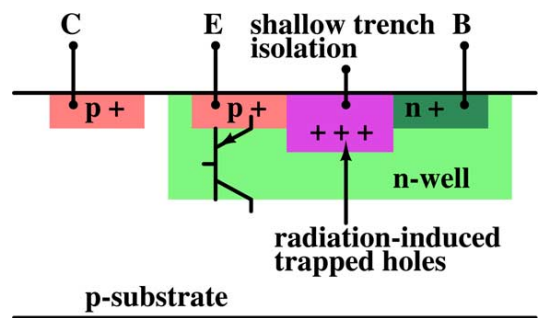

Fig. 2. A pnp transistor formed in a n-well CMOS technology.

summing the proportional to absolute temperature (PTAT) current $I_{R}$ and the complementary to absolute temperature (CTAT) current $I_{R 2}$. By applying this current to a reference resistor, a sub-1-V bandgap reference voltage can be obtained. Assuming M1, M2 and M3 have the same size, we note that $I_{D, M 1}=I_{D, M 2}=I_{D, M 3}\left(I_{D, M x}\right.$ is the drain current of transistor Mx), and $V_{X}=V_{Y}$ due to the negative feedback. The area of diode Q2 is $n$ times that of Q1. Therefore,

$$
\begin{aligned}
V R E F & =\text { Rref } \cdot I_{D, M 3} \\
& =\operatorname{Rref} \cdot\left(\frac{V_{Y}-V_{E B, Q 2}}{R}+\frac{V_{Y}}{R 2}\right) \\
& =\operatorname{Rref} \cdot\left(\frac{V_{E B, Q 1}-V_{E B, Q 2}}{R}+\frac{V_{E B, Q 1}}{R 2}\right) \\
& =\operatorname{Rref} \cdot\left(\frac{V_{T} \ln \frac{I_{D, M 1}}{I_{S S}}-V_{T} \ln \frac{I_{D, M 2}}{n \cdot I_{S S}}}{R}+\frac{V_{E B, Q 1}}{R 2}\right) \\
& =\operatorname{Rref} \cdot\left(\frac{V_{T} \ln n}{R}+\frac{V_{E B, Q 1}}{R 2}\right) .
\end{aligned}
$$

The two diodes are formed by shortening the base and collector of two $p n p$ transistors. In n-well CMOS processes, a pnp transistor can be obtained by doping a $\mathrm{p}^{+}$region inside an $n$-well which serves as the emitter while the n-well itself serves as base, as shown in Fig. 2. The p-type substrate acts as the collector and is inevitably connected to the most negative supply.

\section{B. TID Effects in CMOS Diodes}

Although CMOS gate transistors fabricated in deep-sub-micron technology have shown excellent radiation tolerance, the diode still suffers from radiation induced leakage current [6]. A shallow trench isolation field oxide layer is usually placed surrounding the $\mathrm{p}^{+}$diffusion region, which is the emitter of the $p n p$ transistor. Irradiation induced holes get trapped in the body of the field oxide near the $\mathrm{SiO}_{2}-\mathrm{Si}$ interface. This will increase the base leakage current, and degrade the current gain of the bipolar transistor. Consequently, when the bipolar transistors are used in a bandgap voltage reference, the output voltage/current will undergo dramatic changes.

An explanation of this phenomenon can be obtained by analyzing the conventional bandgap reference circuit in Fig. 1, represented in Fig. 3. When the same small irradiation induced leakage current $\Delta I_{B}$ appears at the emitter-base junction of all diodes, the increased emitter currents in the two branches are different, due to the non-identical numbers of diodes. The increased base leakage current in the left diode is neglected since there is only one unit cell in that branch.

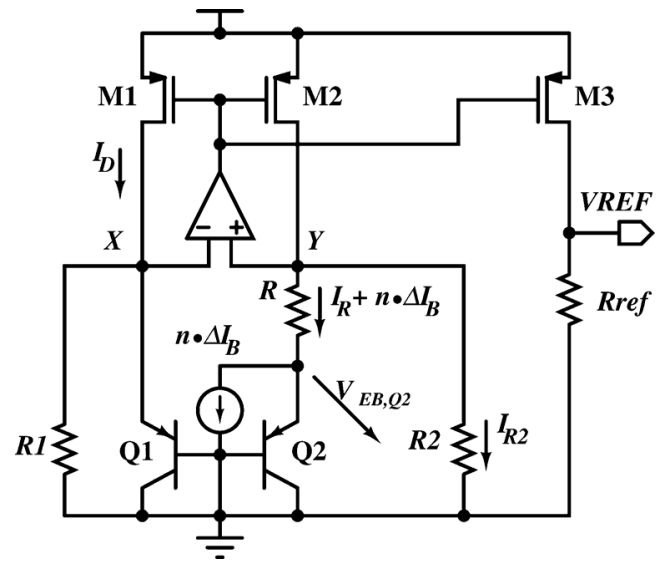

Fig. 3. Modeled TID effects in the conventional bandgap reference as diode base leakage currents.

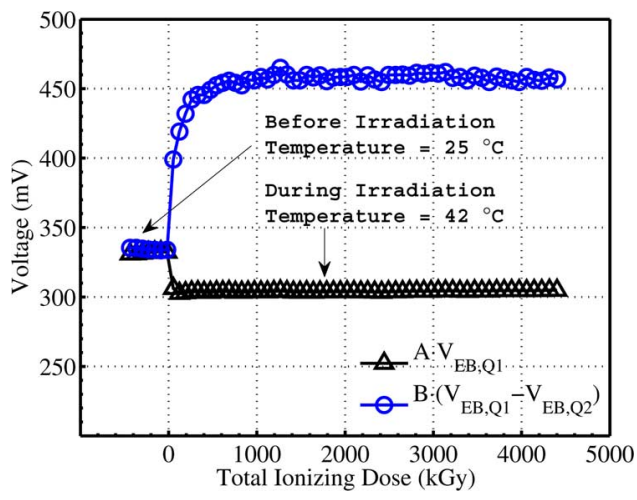

Fig. 4. Measured TID effects in the conventional bandgap reference.

Assuming the collector currents $I_{C}$ remain the same for both diodes after irradiation [6], the emitter-base voltage $V_{E B}$ (the emitter-base voltage of the diode) for both diodes should remain unchanged since $V_{E B}=V_{T} \cdot \ln \left(I_{C} / I_{S S}\right)$. However, the emitter current of the right diode, $I_{R}$, which is equal to $\left(V_{E B, Q 1}-V_{E B, Q 2}\right) / R$, will affect $V_{E B, Q 2}$. The right diode consists of 38 unit cells in this design. Therefore a significant base leakage current will appear in Q2 due to irradiation. Consequently $I_{R}$ has to increase by the same amount to provide this leakage current. This will pull down $V_{E B, Q 2}$, when $V_{E B, Q 1}$ keeps unchanged. Accordingly, $I_{C, Q 2}$ will also decrease until a new balance point is reached. The bandgap voltage

$$
\begin{aligned}
V R E F= & \operatorname{Rref} \cdot\left(I_{R}+I_{R 2}\right) \\
= & \operatorname{Rref} \cdot\left(I_{C, Q 2}+I_{B, Q 2}+\frac{V_{E B, Q 1}}{R 2}\right) \\
= & \operatorname{Rref} \cdot\left(I_{C, Q 2}^{0}-\Delta I_{C, Q 2}+I_{B, Q 2}^{0}+\Delta I_{B, Q 2}\right) \\
& +\operatorname{Rref} \cdot \frac{V_{E B, Q 1}}{R 2},
\end{aligned}
$$

where $I_{C, Q 2}^{0}$ and $I_{B, Q 2}^{0}$ are the initial collector and base currents of the diode, $\Delta I_{B, Q 2}$ is the radiation introduced base leakage current, and $\Delta I_{C, Q 2}$ is the reduced collector current due to the $V_{E B, Q 2}$ drop.

From (2), one can predict that the reference voltage will increase more rapidly at the beginning of the irradiation, when $\Delta I_{B, Q 2}$ is dominant over $\Delta I_{C, Q 2}$. As irradiation continues, 


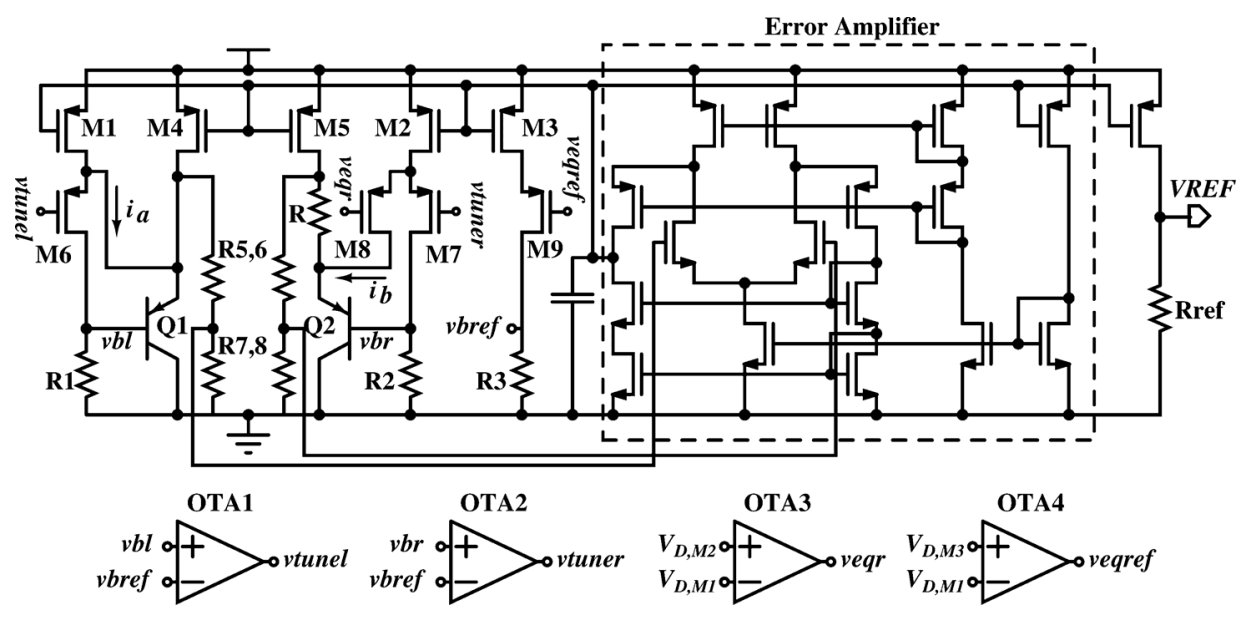

Fig. 5. Schematic of a radiation-hardened CMOS bandgap reference using DBLC.

$V_{E B, Q 2}$ drops further, $\Delta I_{C, Q 2}$ will become larger. Then it compensates parts of the base leakage current, and smoothes the output reference voltage.

Fig. 4 shows the emitter-base voltages of the two diodes in a conventional bandgap reference during a gamma radiation experiment. As derived in (1), by substituting Rref/R2 and Rref $/ R$ with $A$ and $B$, the bandgap reference voltage can be rewritten as

$$
V R E F=A \cdot V_{E B, Q 1}+B \cdot\left(V_{E B, Q 1}-V_{E B, Q 2}\right) .
$$

In order to obtain a temperature/radiation-independent bandgap reference voltage, the average value of two components on the right side of (3) has to remain constant. However, in the case of the conventional bandgap reference, a significant drop is found on $V_{E B, Q 2}$ while $V_{E B, Q 1}$ only has a small shift at the beginning of the irradiation mainly due to a temperature change. Consequently, the output voltage of the bandgap reference increases with the accumulated radiation dose, which is consistent with the theory stated above.

\section{RADIATION-HARDENED BANDGAP REFERENCES}

\section{A. Dynamic Base Leakage Compensation Technique}

A previously reported radiation-hardened bandgap reference based on dynamic-threshold-voltage MOSFET diodes (DTMOS) was proposed in [7] to solve the diodes leakage problem. It replaces the traditional CMOS diodes by DTMOS diodes, which have better radiation hardness. It exhibits good radiation tolerance up to $0.4 \mathrm{MGy}$. However, for applications in the International Thermonuclear Experimental Reactor (ITER), a total dose radiation tolerance more than $1 \mathrm{MGy}$ is required. The DTMOS diodes are sensitive to high levels of total ionizing dose due to threshold variations affecting diode matching. This is because they are actually PMOS transistors operating in the sub-threshold range which are very susceptible to TID effects [8]. Moreover, DTMOS diodes are not standard devices, which make it more difficult to simulate and evaluate the circuit's performance.
In this work, a dynamic compensation technique is proposed to improve the radiation hardness of the conventional bandgap reference where only normal CMOS diodes are used. The schematic is shown in Fig. 5. The purpose of the dynamic compensation unit is to provide all the base current for the diodes. Therefore, only the collector current is flowing in the core circuits. According to early irradiation assessment results [6], the collector current of the diode is nearly unaffected by the increasing ionizing dose. The base leakage currents induced by irradiation will only flow through the compensation circuits, which keeps the bandgap current $I_{D, M 4}$ and $I_{D, M 5}$ remaining constant.

The concept of using feedback compensation circuits to protect sensitive components in a system is similar to the sensitive node active charge cancellation (SNACC) technique presented in [9]. However, the SNACC technique is useful for single-event mitigation, when the sensitive transistors can be layouted closely in a common-centroid fashion to enable differential charge cancellation [10]. For TID effects mitigation, SNACC is less suitable, since the irradiation induced leakage current in different devices will only be the same when they have the same characteristic and the same biasing condition. Moreover, for bandgap reference application, a large number of diodes are normally required and they consume a large area. It is impractical to implement differential protective circuitry for each diode as it brings too much area penalty and layouting difficulties. By contrast, the DBLC technique compensates the leakage current produced in the core circuit globally, and it does not need replica devices. This simplifies the design and layout of the core circuitry and can compensate the TID radiation induced leakage current in CMOS diodes more accurately.

\section{B. Circuits Description}

The core of the radiation-hardened bandgap voltage reference remains the same as in the conventional architecture. The feedback error amplifier is implemented in a two-stage folded-cascode structure. It has a gain of $70 \mathrm{~dB}$ and a phase margin of $60^{\circ}$, when the power consumption is only $4.8 \mu \mathrm{W}$.

The compensation unit works as follows. Resistors R1, R2 and $\mathrm{R} 3$ have the same size. OTA1 and OTA2 are used to keep 


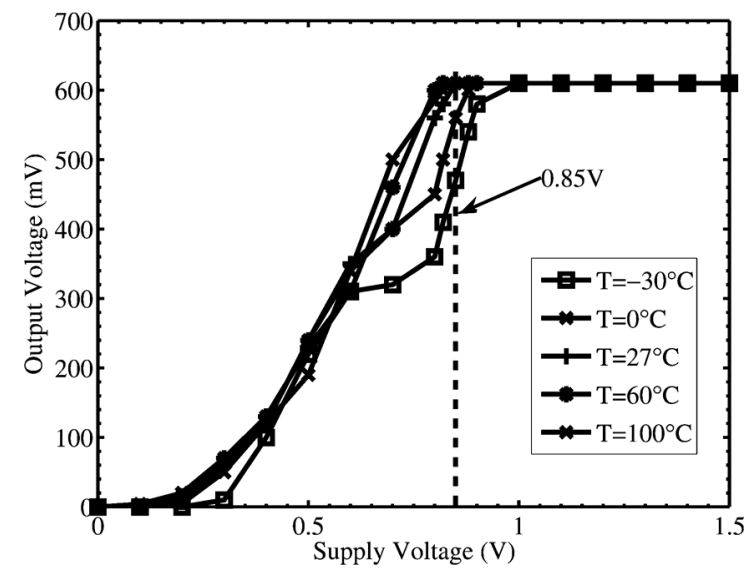

Fig. 6. Output voltage versus supply voltage for different temperature.

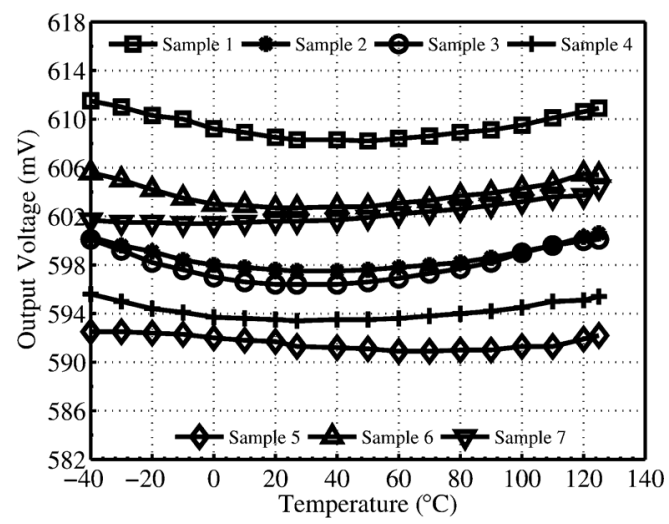

Fig. 7. Measured output voltages versus temperature of 7 different samples.

$v b l=v b r=$ vbref. This is important because $\Delta V_{E, Q 1,2}$ is equal to $\triangle V_{E B, Q 1,2}$ only when $V_{B, Q 1}$ equals $V_{B, Q 2}$, where $\Delta V_{E, Q 1,2}$ and $\Delta V_{E B, Q 1,2}$ are variations of the emitter voltage and the emitter-base voltage of $\mathrm{Q} 1 / \mathrm{Q} 2$, respectively. Therefore, $I_{R 1}=I_{R 2}=I_{R 3}$. Meanwhile, M1, M2 and M3 have the same size, which gives $I_{D, M 1}=I_{D, M 2}=I_{D, M 3}=I_{R 3}$. We also have $I_{R 1}=I_{D, M 1}=I_{D, M 6}+I_{B, Q 1}=I_{D, M 6}+i_{a} . I_{B, Q 1}$ is thus equal to $i_{a}$. For the same principle, $I_{B, Q 2}$ also equals $i_{b}$. When the base current of the diode increases due to the radiation induced leakage, it will be compensated dynamically by this base current servo loop. Transistors M8, M9 and amplifiers OTA3, OTA4 are added to further ensure the currents flowing through M1, M2 and M3 are equal.

\section{EXPERIMENT RESULTS}

The radiation hardened bandgap reference is implemented in a standard $0.13 \mu \mathrm{m}$ CMOS technology, and it consumes $50 \mu \mathrm{A}$ ( $25 \mu \mathrm{A}$ by the bandgap core, and another $25 \mu \mathrm{A}$ by the base leakage compensation circuits.). The core of the reference circuit occupies an area of only $0.056 \mathrm{~mm}^{2}$, and the total area is $0.7 \mathrm{~mm}^{2}$ including all pads. The output voltage of the bandgap reference is designed to be $600 \mathrm{mV}$. The chip is fully functional within a wide supply voltage range of 0.85 to $1.5 \mathrm{~V}$, observed in Fig. 6.

The measured best temperature coefficient (TC) of the bandgap reference is $15 \mathrm{ppm} /{ }^{\circ} \mathrm{C}$ in a range of $-40^{\circ} \mathrm{C}$ to 125 ${ }^{\circ} \mathrm{C}$, as shown in Fig. 7. From 0 to $100^{\circ} \mathrm{C}$, the voltage variation

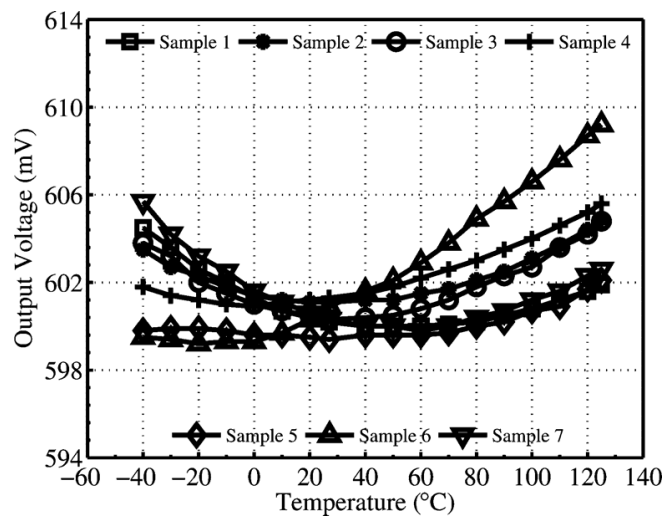

Fig. 8. Trimmed output voltages of 7 different samples.

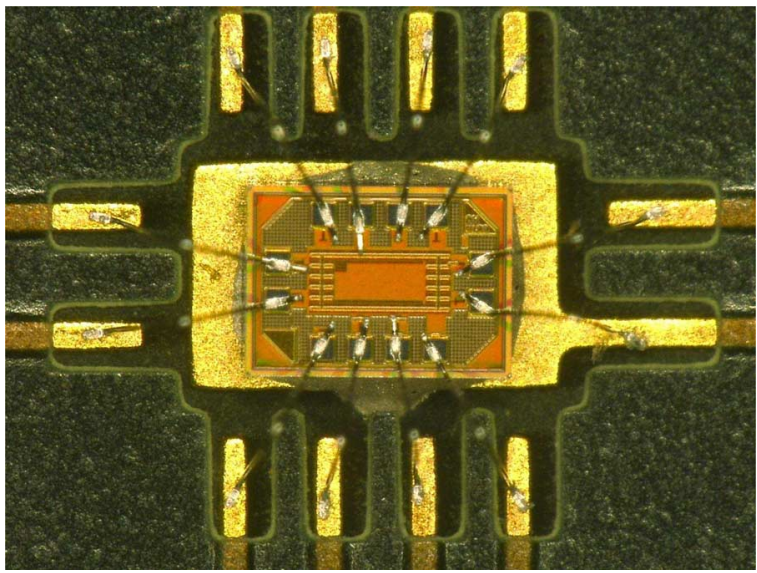

Fig. 9. Die photo of the bonded DBLC bandgap reference circuit after irradiation.

is only $\pm 1 \mathrm{mV}$. A spread of $\pm 15 \mathrm{mV}$ is found in measurement due to process variation. However, this can be trimmed by varying the on-chip programmable resistor. Fig. 8 presents the output voltages of the same samples used in the previous measurement after digital trimming. The voltage spread is reduced to within $\pm 4 \mathrm{mV}$.

More interestingly, the effectiveness of the DBLC technique has also been evaluated by a real-time gamma irradiation assessment. The experiment was carried out from the "Brigitte" facility at the Belgium Nuclear Research Centre, SCK-CEN. Substrates bonded with both conventional bandgap references and radiation-hardened bandgap references (shown in Fig. 9) from the same technology run are irradiated with ${ }^{60} \mathrm{Co}$ gamma source. A high dose rate of $27 \mathrm{kGy} / \mathrm{h}$ is obtained, which enables us to achieve a TID of $4.5 \mathrm{MGy}$ in one week.

Fig. 10 shows the emitter-base voltages of two diodes in the radiation-hardened bandgap reference. Thanks to the dynamic base leakage compensation technique, both $V_{E B, Q 1}$ and $V_{E B, Q 2}$ are immune to the total ionizing dose, where only shifts caused by temperature drift are found. As a result, the output reference voltage of the bandgap employing the DBLC technique stays equal to the pre-rad value. Output voltages of the radiation-hardened bandgap reference and the conventional bandgap reference of one measured sample are shown in Fig. 11. A variation of only $1 \%$ is found from 0 


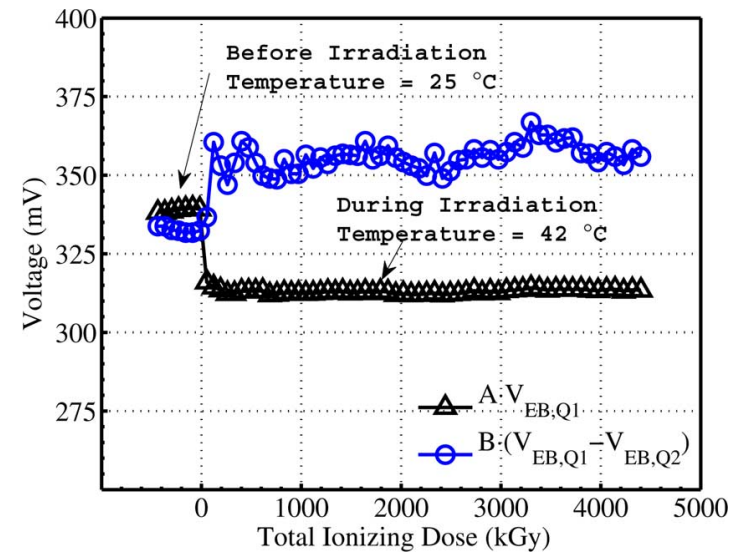

Fig. 10. Emitter-base voltages of the diodes in the radiation-hardened bandgap reference using DBLC.

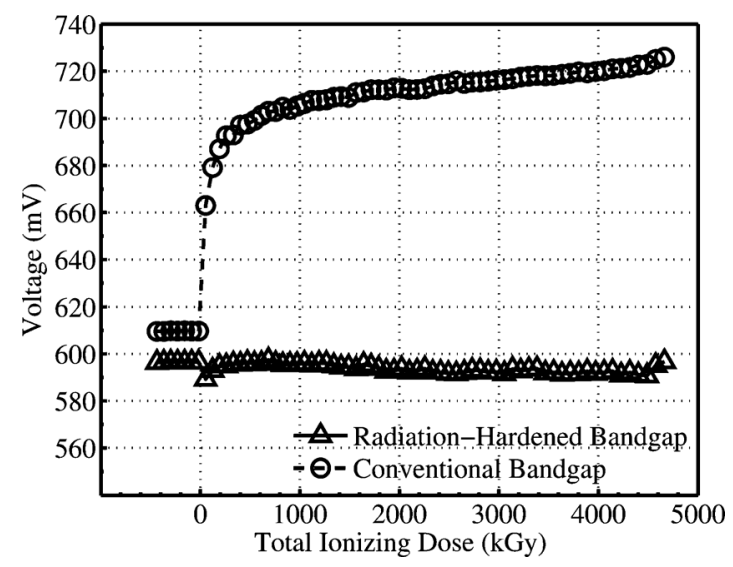

Fig. 11. Measured output voltages of the radiation-hardened bandgap reference and the conventional bandgap reference.

to 4.5 MGy. A small dip is observable at the beginning of the irradiation. This is due to the latency existed in the base leakage compensation network which requires a longer time to provide the significantly increased base current, after all diodes are irradiated instantly at such a high radiation dose rate. On the other hand, the output voltage of the conventional bandgap reference increases more than $15 \%$ during irradiation. After the irradiation stopped, the output voltage of the DBLC bandgap reference can be easily recovered to the pre-rad value by self-annealing.

More than one sample has been measured during the irradiation. The upper group of voltage curves in Fig. 12 is obtained from 5 different conventional bandgap reference samples. They show an average increase of $17 \%$ after irradiation. The lower group of voltage curves which exhibits a variation of less than $3 \%$ is obtained from 5 DBLC bandgap samples. A closed-in inspection of this variation is shown in Fig. 13. Some samples show a slowly increasing slope or decreasing slope in the output reference voltage, which is mainly due to the matching imperfection in the base leakage compensation network.

\section{CONCLUSION}

This work presents a radiation-hardened CMOS bandgap voltage reference. It provides a reference voltage of $600 \mathrm{mV}$

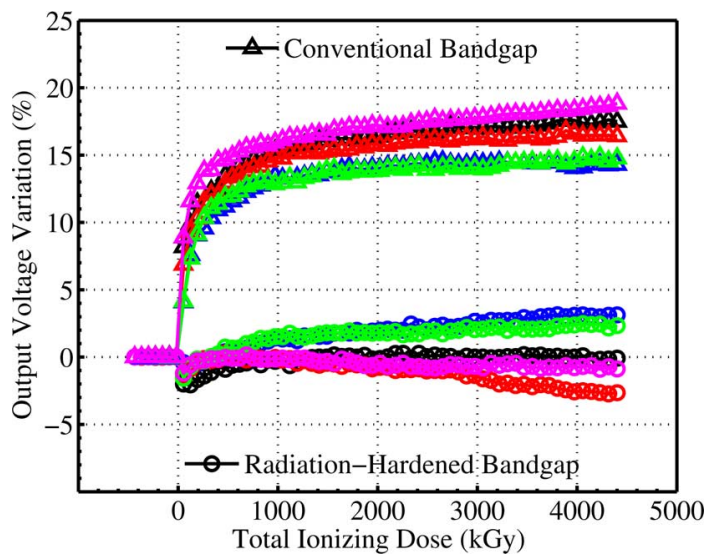

Fig. 12. Output voltage variation of 5 conventional bandgap references and 5 radiation-hardened bandgap references.

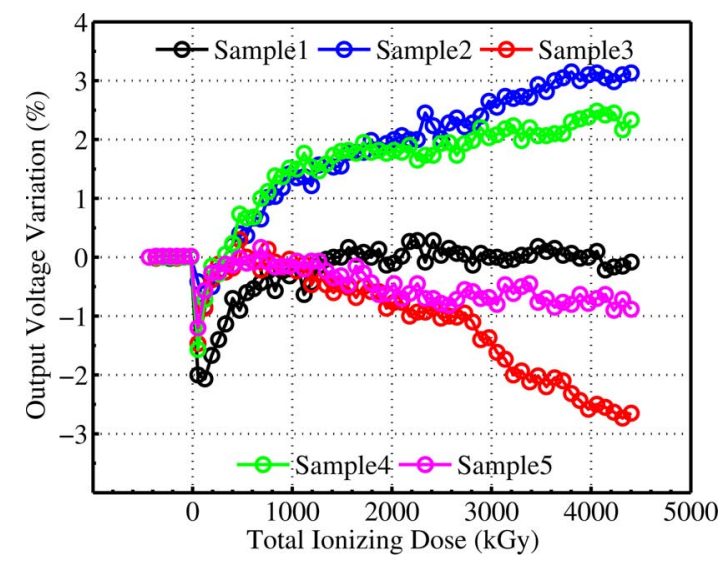

Fig. 13. Closed-in inspection of output voltage variation of the DBLC radiation-hardened bandgap references.

TABLE I

MEASURED SPECIFICATIONS

\begin{tabular}{|c|c|}
\hline Technology & $0.13 \mu \mathrm{m}$ CMOS \\
\hline Area & $0.056 \mathrm{~mm}^{2}$ (core) \\
$0.7 \mathrm{~mm}^{2}$ (chip)
\end{tabular}

under room temperature. The bandgap voltage-temperature curve exhibits a TC of $15 \mathrm{ppm} /{ }^{\circ} \mathrm{C}$ from $-40^{\circ} \mathrm{C}$ to $125^{\circ} \mathrm{C}$, and the voltage variation from $0^{\circ} \mathrm{C}$ to $100^{\circ} \mathrm{C}$ is only $\pm 1 \mathrm{mV}$. The bandgap reference is also compatible with sub-1 V operation. A real-time gamma irradiation assessment with a dose rate of $27 \mathrm{kGy} / \mathrm{h}$ has been performed to verify the bandgap reference's TID tolerance. The output voltage of the bandgap reference 
using the DBLC technique has shown a variation of only $3 \%$ after 4.5 MGy.

The measured specifications of the DBLC bandgap reference are summarized in Table I.

\section{REFERENCES}

[1] K. E. Kujik, "A precision reference voltage source," IEEE J. SolidState Circuits, vol. 8, pp. 222-226, Jun. 1973.

[2] W. Snoeys, G. Anelli, and M. Campbell et al., "Integrated circuits for particle physics experiments," IEEE J. Solid-State Circuits, vol. 35, no. 12, pp. 2018-2030, Dec. 2000.

[3] R. C. Lacoe, "Improving integrated circuit performance through the application of hardness-by-design methodology," IEEE Trans. Nucl. Sci., vol. 55, no. 4, pp. 1903-1925, Aug. 2008.

[4] P. Moreira, Radiation Effects on the "CERN_Bandgap" Circuit [Online]. Available: http://proj-qpll.web.cern.ch/proj-qpll/images/bandgapRadEffects.pdf Feb. 2004
[5] H. Banba, H. Shiga, and A. Umezawaand et al., "A CMOS Bandgap reference circuit with Sub-1-V operation," IEEE J. Solid-State Circuits, vol. 34, no. 5, pp. 670-674, May 1999.

[6] R. L. Pease, "Total ionizing dose effects in bipolar devices and circuits," IEEE Trans. Nucl. Sci., vol. 50, no. 3, pp. 539-551, Jun. 2003.

[7] V. Gromov, A. J. Annema, R. Kluit, and J. L. Visschers, "A radiation hard bandgap reference circuit in a standard $0.13 \mu \mathrm{m}$ CMOS technology," IEEE Trans. Nucl. Sci., vol. 54, no. 6, pp. 2727-2733, Dec. 2007.

[8] F. Faccio and G. Cervelli, "Radiation-induced edge effects in deep submicron CMOS transistors," IEEE Trans. Nucl. Sci., vol. 52, no. 6, pp. 2413-2420, Dec. 2005.

[9] R. W. Blaine, S. E. Armstrong, and J. S. Kauppila et al., "RHBD bias circuits utilizing sensitive node active charge cancellation," IIEEE Trans. Nucl. Sci., vol. 58, no. 6, pp. 3060-3066, Dec. 2011.

[10] S. E. Armstrong, B. D. Olson, and W. T. Holman et al., "Demonstration of a differential layout solution for improved ASET tolerance in CMOS A/MS circuits," IEEE Trans. Nucl. Sci., vol. 57, no. 6, pp. 3615-3619, Dec. 2010. 